\title{
Uma abordagem didática da natureza dos processos de aquecimento da atmosfera estelar
}

(A pedagogical approach of the nature of the heating processes in the stellar atmosphere)

\author{
Osman Rosso Nelson ${ }^{\boxplus}$ \\ Departamento de Física Teórica e Experimental, Universidade Federal do Rio Grande do Norte, Natal, RN, Brasil
}

Recebido em 14/1/2013; Aceito em 14/3/2013; Publicado em 26/9/2013

O presente trabalho pretende, através de uma abordagem didática, resgatar a íntima ligação entre processos de propagação de ondas no plasma estelar e aquecimento coronal.

Palavras-chave: aquecimento estelar, ondas sonoras, ondas magnetossônicas, ondas Alfvén.

\begin{abstract}
Using a pedagogical approach, this work aims at rescuing the intimate connection between the processes of wave propagation in plasma and the stellar coronal heating.

Keywords: stellar heating, sound waves, magnetosonic waves, Alfvén waves.
\end{abstract}

\section{Introdução}

O plasma, hoje conhecido como o quarto estado da matéria, foi identificado primeiramente (1879) por Sir. William Crookes em um tubo, tubo de Crookes, e inicialmente denominado de matéria radiante. A natureza dessa matéria radiante foi estudada por Sir. J.J. Thomson em 1897. Posteriormente, em 1927, Irving Langmuir designou de plasma. O plasma, constituinte essencial do universo, é um estado físico da matéria que se assemelha a um gás com grande número de partículas ionizadas. Essa presença de portadores de carga dá ao meio a característica de um condutor, portanto, capaz de responder a campos eletromagnéticos. Esse meio condutor exibe neutralidade macroscópica e seus elementos constitutivos apresentam comportamento coletivo devido às forças coulombianas de longo alcance. Um modelo simplificado para descrevê-lo, que adotaremos aqui, é o da magnetohidrodinâmica.

Na magnetohidrodinâmica, o plasma é tratado como um único fluido, contendo um formalismo que comporta as equações de conservação de massa, momentum e energia, uma lei de Ohm generalizada e as equações de Maxwell. Nesse contexto, objetivamos descrever, de forma didática, o intrigante processo de aquecimento coronal, sinalizando para a importância dos processos de transporte de energia associados às ondas em plasma. Como veremos, o plasma estelar, estando nor-

\footnotetext{
${ }^{1}$ E-mail: osman@dfte.ufrn.br.

malmente permeado por campos magnéticos, torna-se um ambiente natural para propagação de diversos tipos de ondas, como as que serão descritas posteriormente (sonoras, magnetosônicas e Alfvén). Esse artigo aborda o problema do aquecimento da atmosfera estelar numa linguagem que permite a inclusão dos leitores não familiarizados com astrofísica. Um grande esforço foi feito para agregar, num só espaço, formas didáticas de apresentar todas as ferramentas necessárias à compreensão dos processos envolvidos. Tal esforço contempla o uso, sempre que possível, da força da analogia como instrumento didático, bem como, tenta apresentar, de forma detalhada, os desenvolvimentos matemáticos inerentes ao assunto. Uma contribuição extra à compreensão do processo de aquecimento é dada na seção 5 . Nessa seção, o leitor terá a oportunidade de ver quais parâmetros tornam-se relevante para o amortecimento dos diversos tipos de ondas e, com isso, mesmo dentro das limitações teóricas do desenvolvimento apresentado, poder inferir quais mecanismos podem ser mais eficientes dependendo do tipo de ambiente estelar. Assim, acredito contribuir, principalmente, com os estudantes de iniciação científica que, normalmente, esbarram em textos muito especializados.

Ressalto, ainda, que parte desse esforço está ancorada no trabalho desenvolvido durante a tese de doutorado do autor [1]. 


\section{Situando o problema do aquecimento da corona estelar}

\subsection{Atmosfera estelar}

A análise estrutural de uma estrela permite distinguir duas grandes regiões: o interior estelar e a atmosfera. O interior estelar é composto por uma região central, núcleo, um envoltório radiativo e um envoltório convectivo, enquanto a atmosfera estelar encontra-se subdividida nas regiões designadas por: fotosfera (esfera de luz), cromosfera (esfera colorida) e coroa (a raiz latina da palavra corona), constituindo um refinado laboratório de física de plasma, onde estruturas complexas e variáveis estão presentes. Nosso objeto de estudo, a atmosfera, começa por uma camada mais interna, a fotosfera, cuja base define o que usualmente se chama de superfície da estrela, que serve de referência para as medidas de alturas na atmosfera.

Muito embora as estrelas, em diferentes estágios evolutivos, exibam características peculiares, as observações detalhadas do Sol, facilitadas pela sua proximidade com nosso planeta, têm fornecido subsídio para ampliar de forma significativa nossa compreensão das estrelas em geral. Antes de generalizar a discussão sobre a atmosfera estelar, breves considerações sobre a atmosfera solar serão feitas. A Fig. 1 ilustra a estrutura do Sol.

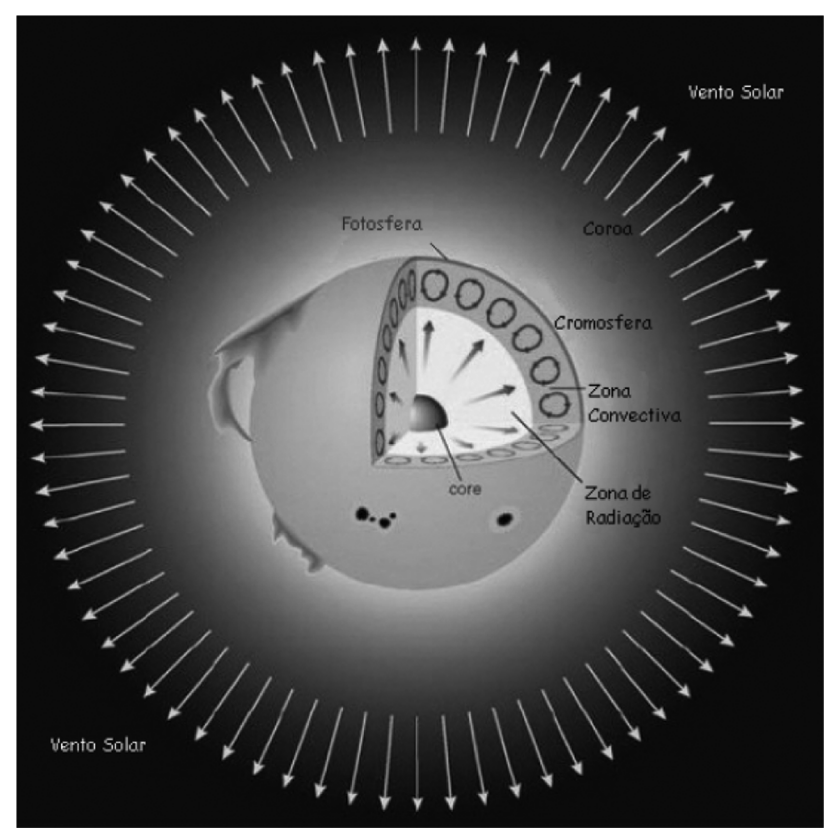

Figura 1 - Regiões do Sol: representação do interior e da atmosfera solar marcada com a presença de estruturas como: manchas solares, proeminências, vento solar. Fonte: adaptação da Ref. [2].

\subsection{Atmosfera solar}

A primeira camada da atmosfera solar, fotosfera, exibe uma profundidade em torno de $400 \mathrm{~km}$, seguida da cromosfera com aproximadamente $2000 \mathrm{~km}$, de uma região de transição estreita e, finalmente, de uma extensa coroa, cuja espessura não é bem definida, mas chega a vários raios solares.

Na Fig. 2 vê-se uma ilustração simplificada do comportamento térmico dessas camadas do Sol. Apesar de não abranger toda extensão da coroa, pode-se ter uma idéia relativamente ampla do comportamento da densidade e da temperatura solar. Essas informações vêm auxiliar a compreensão das características básicas das diferentes camadas, bem como do intrigante comportamento térmico das camadas mais externas e revelar o problema central do aquecimento atmosférico estelar.

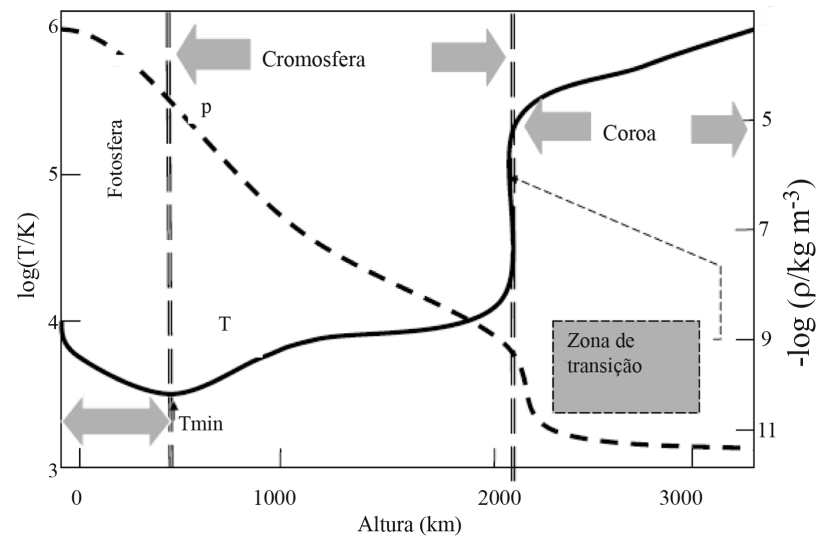

Figura 2 - Camadas Atmosféricas do Sol. Fonte: adaptação da Ref. [3].

Na Fig. 2 notamos, à medida que nos distanciamos do interior do Sol, uma diminuição contínua da densidade da atmosfera. Ressalta-se também que, na fotosfera, a temperatura diminui até atingir um valor mínimo e, a partir desse ponto, inicia-se a nova camada, a cromosfera.

Como bem estabelecido, uma elevada quantidade de energia é liberada no interior estelar, devido às reações de fusão. Dessas reações nucleares decorre a transmutação de elementos leves em elementos mais pesados e, consequentemente, uma produção enorme de energia. Essa região mais interna da estrela funciona como uma espécie de fornalha, sendo natural que se espere um decréscimo de temperatura à medida que nos distanciamos do foco de aquecimento. Entretanto, em geral, tal comportamento térmico não ocorre na atmosfera das estrelas. A Fig. 2 mostra que essa expectativa de decréscimo da temperatura é satisfeita apenas na fotosfera. Uma simples análise da Fig. 2 revela, a partir do ponto chamado de Tmín, que a temperatura cresce de forma contínua e relativamente suave até um ponto onde ocorre um crescimento abrupto, demarcando a chamada zona de transição. Da zona de transição em diante começa a camada mais externa da atmosfera, com temperaturas ainda mais elevadas. Estamos falando da coroa solar.

Nesse ponto da nossa abordagem, surge a inquietante questão: como justificar essas altas temperaturas? Elucidar as causas da existência de altas temperaturas 
nas camadas mais externas da atmosfera do Sol ou de uma estrela em geral tem sido uma tarefa desafiadora. Até o presente momento, apesar de várias propostas de mecanismos de aquecimentos terem sido feitas, o problema do aquecimento das camadas mais externas continua em aberto.

Sabemos que praticamente todas as estrelas estão envolvidas por uma camada quente, onde a temperatura alcança valores quase tão altos quanto os valores presentes nos núcleos estelares. Essas camadas aquecidas são responsáveis pela emissão de raio-X, UV (radiação ultravioleta) e emissões de rádio.

\subsection{Mecanismos de aquecimento da atmosfera estelar}

Tanto as observações realizadas em terra como as obtidas por satélite têm mostrado que, provavelmente, todas as estrelas, com possível exceção das estrelas A, têm regiões de suas atmosferas com temperatura muito maior que o valor fotosférico. Para leitores menos familiarizados com a classificação estelar, devo ressaltar que uma estrela A se encontra na região do diagrama HR, principal diagrama destinado ao estudo de evolução estelar, na região onde se encontram aquelas com altas temperaturas. Esse diagrama HR (Hertzsprung e Russell) faz a classificação das estrelas com sendo tipos espectrais O, B, A, F, G, K, M. Nessa classificação a temperatura decresce de O para M. O Sol, por exemplo, uma estrela do tipo espectral G, por ser nossa estrela mais próxima, oferece um nível de informação mais amplo das estruturas que são exibidas por suas diferentes camadas. Edlén [4] descobriu que a corona solar é uma camada gasosa com temperatura de milhões de graus. Desde muitos anos temos evidências de que, próximo da superfície visível do Sol, o gradiente de temperatura torna-se zero e que a temperatura da cromosfera e da corona cresce radialmente para fora da região central da estrela. Historicamente, a explicação desse fenômeno nasceu das iniciativas independentes de Biermann [5, 6] e Schwarzschild [7]. Eles sedimentaram suas explicações nos processos de aquecimentos originados por dissipação de ondas de choques. Tais distúrbios seriam gerados por ondas sonoras de pequenas amplitudes na zona convectiva de hidrogênio e se propagariam em direção às camadas mais externas. Resumidamente, tem-se o crescimento em amplitude, como consequência do decréscimo de densidade das camadas exteriores, a acumulação para o choque e a rápida dissipação da energia. A parcela de energia solar carregada pelas ondas (transportada pela fotosfera e zona de transição) é liberada na cromosfera e coroa solar. O balanço de energia, nessas camadas exteriores, entre a energia fornecida pelas ondas e a energia emitida em forma de radiação dá o perfil de temperatura peculiar dessas camadas.

Essas idéias foram elaboradas de forma detalhada por Schatzman [8] e por Schirmer [9], mostrando que muitas das características observadas da cromosfera e da corona solar podiam ser entendidas através de suas concepções. Diversos autores [10-29], posteriormente, desenvolveram essa idéia inicial.

Em adição a essa atraente proposta de aquecimento, Alfvén [30] apresentou um mecanismo semelhante de aquecimento para a alta atmosfera estelar, diferenciando-o do mecanismo anterior pelo tipo de onda envolvido no processo. Ao invés de ondas mecânicas, ondas sonoras, ele propôs ondas que surgem de perturbações no campo magnético conhecidas como ondas Alfvén. Essas ondas passaram a ser consideradas como agentes capazes de aquecer a alta atmosfera estelar. A idéia de Alfvén, posteriormente respaldada por diversos autores [31-33], dá ênfase ao fato da atmosfera solar ser um gás parcialmente ionizado e que se encontra imerso num campo magnético. Do movimento desse plasma congelado ao campo magnético, nascem as ondas Alfvén, que se propagam e são dissipadas na atmosfera estelar mais externa. Evidências mais diretas da veracidade dessa concepção são fornecidas pelos resultados observacionais [34-36], em que praias (plages) de cálcio, regiões onde a linha $\mathrm{K}$ de emissão cromosférica de Ca II é excepcionalmente brilhante, coincidem com regiões de fortes campos magnéticos. O maior aquecimento nessas regiões plages, está aparentemente relacionado à presença desses fortes campos magnéticos.

Outra indicação da importância do campo magnético no aquecimento das camadas exteriores do Sol é fornecida pelas espículas [37] ou jatos [38], observados no limbo cromosférico superior. Essas características foram consideradas por muitos autores [31, 39] como exemplos visíveis das ondas de choques que se propagam para fora da cromosfera e vão se dissipar na corona. Assim, parece existir uma conexão entre campo magnético solar e os distúrbios propagando-se para fora na cromosfera superior. Outra observação mais indireta ressaltando a importância do campo magnético é fornecida pelas observações das linhas de emissão K do espectro de estrelas tipo tardio. Para essas estrelas, a estrutura de suas superfícies não pode ser resolvida. Apesar disso, parece claro que as linhas de emissão devem surgir na cromosfera exterior, como no Sol.

É claro que, do ponto de vista teórico, os efeitos magnetohidrodinâmicos devem ampliar sua importância em alguma altura da cromosfera, porque, enquanto em primeira aproximação, a velocidade do som $\left(V_{S}\right)$ é constante, a velocidade das ondas Alfvén $\left(V_{A}\right)$ cresce rapidamente para o exterior devido ao decréscimo da densidade da atmosfera, Eq. (ए2). Dessa forma, o campo magnético interfere pouco, quando $V_{A}<V_{S}$, mas não pode ser negligenciado, quando $V_{A}>V_{S}$.

De maneira geral, sabemos que as camadas exteriores da atmosfera numa estrela são caracterizadas por grandes perdas de energia, como as observadas na cro- 
mosfera (predominantemente perdas por radiação) e as diversas perdas observadas na corona estelar (tanto por condução, radiação, como por fluxo de vento estelar). Portanto, se não existissem mecanismos capazes de fornecer constantemente energia a essas camadas, elas rapidamente esfriariam. Desse entendimento, surge uma série de propostas de mecanismos de aquecimento para as camadas exteriores da atmosfera estelar. Uma ampla visão dessas propostas de aquecimento da cromosfera e corona estelar pode ser encontrada nos seguintes artigos de revisão: [12, 17, 31, 40-72]. Uma reflexão cuidadosa nos permite concluir que, provavelmente, o aquecimento da cromosfera e corona estelar não pode estar reduzido a um único processo, contemplando, assim, inúmeros mecanismos ao mesmo tempo. Alguns desses mecanismos podem operar globalmente, enquanto outros apenas em situações físicas particulares. Nesse sentido, apresentaremos, nas sessões seguintes, uma discussão didática dos tipos de ondas, já mencionados, que, provavelmente, são responsáveis pelo perfil do aquecimento coronal nas diferentes escalas local ou global.

\section{Ondas acústicas, ondas Alfvén e magnetosônicas}

\subsection{Introdução}

O plasma estelar enriquecido com o campo magnético é palco de diversos tipos de processos ondulatórios, dentre os quais ressaltam-se: as ondas acústicas, as magnetosônicas e as ondas Alfvén. As ondas acústicas constituem uma forma bem familiar de propagação de energia, que normalmente é analisada no contexto em que o meio é um fluido não-condutor, compressível e livre de campos magnéticos. Começaremos, na perspectiva da hidrodinâmica usual, uma breve discussão sobre as ondas acústicas. Posteriormente serão apresentados outros tipos de ondas que podem surgir num plasma magnetizado dentro de uma abordagem que garanta a força da analogia como instrumento didático. Passo adicional de natureza mais matemática, incluindo todos os tipos de ondas no plasma, poderá ser apreciado na seção 4.2. Nessa seção, a Eq. (55), derivada da estrutura das equações da MHD, conduzirá de forma natural e conveniente aos diversos tipos de ondas que um plasma magnetizado pode suportar.

Tendo em vista que o depósito de energia na atmosfera estelar, via absorção de ondas, constitui um mecanismo bastante citado na literatura especializada, é natural o interesse em descrever esses processos oscilatórios para, assim, poder explicitar os parâmetros que interferem na absorção dessas ondas.

\subsection{Ondas acústicas}

O movimento oscilatório de pequena amplitude num fluido compressível é bem conhecido como ondas sono- ras. Em tal processo, o fluido está sujeito a compressões e rarefações, que serão descritas através de perturbações nos valores de equilíbrio das grandezas que descrevem o fluido. Para distinguir os valores das perturbações dos valores de equilíbrio, utilizaremos, para estes, índice zero, e, para aqueles, uma linha na parte superior da letra. Uma exceção será feita para a perturbação na velocidade, que será designada apenas por v. Portanto, $p_{0}$ e $\rho_{0}$ representam as grandezas pressão e densidade no equilíbrio, enquanto $\mathbf{v}, p^{\prime}$ e $\rho^{\prime}$ representam as perturbações na velocidade, pressão e densidade, respectivamente. Assim, escreveremos

$$
p=p_{0}+p^{\prime} ; \quad \rho=\rho_{0}+\rho^{\prime} .
$$

As variações de pressão e densidade comparadas aos valores de equilíbrio são extremamente pequenas, $p^{\prime}<<p_{0}$ e $\rho^{\prime}<<\rho_{0}$.

Sendo assim, as equações da continuidade e de Euler, a saber

$$
\begin{gathered}
\frac{\partial \rho}{\partial t}+\nabla \cdot(\rho \mathbf{v})=0 \\
\frac{\partial \mathbf{v}}{\partial t}+(\mathbf{v} \cdot \nabla) \mathbf{v}=-\frac{1}{\rho} \nabla p,
\end{gathered}
$$

ficarão reduzidas, após desprezar termos como $(\mathbf{v} \cdot \boldsymbol{\nabla}) \mathbf{v}$. Em outras palavras, uma vez que o processo descrito acima é para pequenas oscilações (pequenas velocidades), essa abordagem permite desprezar termos como $(\mathbf{v} \cdot \boldsymbol{\nabla}) \mathbf{v}$, que aparece na equação de Euler, Eq. (四), bem como os termos de segunda ordem, na equação da continuidade, Eq. (四).

Dessa forma, as equações da continuidade e de Euler serão escritas como

$$
\begin{aligned}
& \frac{\partial \rho^{\prime}}{\partial t}+\rho_{0} \boldsymbol{\nabla} \cdot \mathbf{v}=0 \\
& \frac{\partial \mathbf{v}}{\partial t}+\frac{1}{\rho_{0}} \nabla p^{\prime}=\mathbf{0} .
\end{aligned}
$$

Para uma porção do fluido de massa Mocupando um volume $V$, um acréscimo de pressão $(\Delta p>0)$ provoca, em geral, uma diminuição de volume $(\Delta V<0)$. Define-se, portanto, o módulo de compressibilidade $\kappa$ como sendo

$$
\kappa=-\frac{\Delta V / V}{\Delta p} .
$$

Percebe-se então que, quanto mais compressível for um fluido, maior será a variação percentual do volume provocada por uma dada variação de pressão. Consequentemente, o módulo de compressibilidade $\kappa$ será maior.

Outra grandeza relevante para esse estudo é o módulo de elasticidade, B. O módulo de elasticidade é o inverso da compressibilidade, logo

$$
B=\frac{1}{\kappa} .
$$


Como a densidade volumétrica $(\rho)$ é dada por $\rho=M / V$ e $\Delta \rho=-\rho \Delta V / V$, a Eq. (G) se transforma em

$$
\kappa=\frac{1}{\rho} \frac{\Delta \rho}{\Delta p} .
$$

Portanto, o módulo de elasticidade será dado aproximadamente por

$$
B=\rho_{0} \frac{\Delta p}{\Delta \rho}
$$

ou ainda,

$$
B=\rho_{0} \frac{p^{\prime}}{\rho^{\prime}} .
$$

Assim, da Eq. (四), temos

$$
\rho^{\prime}=\frac{\rho_{0}}{B} p^{\prime}
$$

As Eqs. (四) e (四) relacionam as grandezas perturbadas $p^{\prime}, \rho^{\prime}$ e v. Para a obtenção de equações que expressem a evolução temporal de cada grandeza perturbada, pode-se, inicialmente, eliminar as grandezas $\rho^{\prime}$ e $\mathbf{v}$ e ficar apenas com uma equação para a perturbação na pressão $p^{\prime}$.

Usando a Eq. (四) na Eq. (田), eliminamos $\rho^{\prime}$ e obtemos

$$
\frac{\partial p^{\prime}}{\partial t}=-B \boldsymbol{\nabla} \cdot \mathbf{v} .
$$

Tomando-se a divergência da Eq. (回), eliminamos $\mathbf{v}$, ou seja

$$
\frac{\partial}{\partial t}(\boldsymbol{\nabla} \cdot \mathbf{v})=-\frac{1}{\rho_{0}} \nabla^{2} p^{\prime} .
$$

Derivando-se a Eq. (四) em relação ao tempo, obtém-se

$$
\frac{\partial^{2} p^{\prime}}{\partial t^{2}}=-B \frac{\partial}{\partial t}(\boldsymbol{\nabla} \cdot \mathbf{v}) .
$$

Substituindo-se a Eq. ([4]) na Eq. ([4]), encontramos

$$
\nabla^{2} p^{\prime}-\frac{\rho_{0}}{B} \frac{\partial^{2} p^{\prime}}{\partial t^{2}}=0
$$

ou, ainda,

$$
\nabla^{2} p^{\prime}-\frac{1}{V_{S}^{2}} \frac{\partial^{2} p^{\prime}}{\partial t^{2}}=0 .
$$

A Eq. (며) é uma equação de onda para a perturbação da pressão propagando-se com velocidade

$$
V_{S}=\sqrt{\frac{B}{\rho_{0}}},
$$

onde $V_{S}$ é a velocidade das ondas sonoras.
Seguindo um processo análogo, eliminando-se $p^{\prime}$, encontramos a equação de onda para $\mathbf{v}$, ou seja,

$$
\nabla^{2} \mathbf{v}-\frac{1}{V_{S}^{2}} \frac{\partial^{2} \mathbf{v}}{\partial t^{2}}=\mathbf{0} .
$$

Seguindo agora esses procedimentos aplicados para $\rho$, é fácil mostrar que

$$
\nabla^{2} \rho^{\prime}-\frac{1}{V_{S}^{2}} \frac{\partial^{2} \rho^{\prime}}{\partial t^{2}}=0 .
$$

Para obtermos o comportamento da velocidade do som em função de grandezas como temperatura, $T$, pressão, $p$, densidade do meio etc., façamos uma análise no contexto de um gás ideal. Sabe-se que a equação de estado do gás ideal é dada por

$$
p=\frac{K T}{m} \rho,
$$

onde $K$ é a constante de Boltzmann, $T$ é a temperatura absoluta, $m$ a massa de uma partícula e que, em adição, as vibrações sonoras são rápidas o suficiente para serem consideradas um processo adiabático. Processos adiabáticos num gás ideal satisfazem a equação

$$
p V^{\gamma}=c t e,
$$

sendo $V$ o volume do gás e $\gamma$ a razão entre o calor específico à pressão constante, $C_{P}$, e calor específico a volume constante, $C_{V}$,

$$
\gamma=C_{P} / C_{V} .
$$

Desta forma, temos

$$
p=b \rho^{\gamma},
$$

onde $b$ é uma constante.

Na condição de processo adiabático, a Eq. (四) pode ser escrita como

$$
B=\rho(d p / d \rho)_{S} .
$$

Nessa equação, o índice $s$ denota que o processo é reversível e adiabático.

Logo, da Eq. ([2:3) e da Eq. (펴), obtemos

$$
B=\gamma p .
$$

Portanto, fazendo uso das Eqs. (ㅁ] ) e (四), conseguiremos expressar a velocidade da onda sonora em função das grandezas pressão e densidade, a saber,

$$
V_{S}=\sqrt{\gamma p / \rho} .
$$

Por outro lado, usando a Eq. (四) na Eq. (四), obtemos a velocidade da onda sonora em função da temperatura, a saber,

$$
V_{S}=\sqrt{\gamma K T / m} .
$$


Com boa aproximação, podemos afirmar que

$$
V_{S} \alpha \sqrt{T} \text {. }
$$

\subsection{Ondas Alfvén}

Dentro da perspectiva da hidrodinâmica usual, excluindo as ondas de superfície, as únicas possíveis com pequeno comprimento de onda são as ondas acústicas (ondas compressíveis longitudinais, analisadas na seção anterior).

Como já explicitado no âmbito da magnetohidrodinâmica, um fluido compressível imerso num campo magnético possibilita a existência de outros tipos de movimento ondulatório, tais como as ondas Alfvén e as ondas magnetosônicas.

Uma das propriedades notáveis do campo magnético em MHD é a propagação de ondas transversais ao longo das linhas de indução que representam esse campo. Tal processo muito se assemelha ao obtido quando cordas elásticas são postas para vibrar.

Como bem se conhece, as vibrações transversais numa corda elástica se propagam com uma velocidade dada por

$$
V_{\text {Corda }}=\sqrt{\text { tensão/densidade }} .
$$

Quando um fluido condutor imerso num campo magnético é posto para oscilar, o efeito do congelamento das linhas de campo no fluido faz com que essas linhas também oscilem. Assim, trocando-se as cordas elásticas por linhas de indução magnética, a analogia fica perfeita. Nessa situação, a velocidade da onda gerada será dada de forma totalmente análoga, bastando substituir a tensão da corda pela tensão magnética. Portanto, estão presentes os elementos fundamentais para que um modo oscilatório se propague através do meio: a força restauradora (produzida pelo campo magnético) e a inércia (inerente à densidade do fluido).

Desta forma, levando-se em conta que a tensão magnética $T_{M}$ [73] é dada por

$$
T_{M}=B_{0}^{2} / 4 \pi
$$

a velocidade $V_{A}$ desse novo tipo de ondas será, após substituir o valor da tensão magnética na equação

$$
V_{A}=\sqrt{\text { tensão magnética/densidade }}
$$

$$
V_{A}=\sqrt{B_{0}^{2} / 4 \pi \rho_{0}}
$$

Alfvén (1942) foi o primeiro a assinalar existência dessas ondas, daí serem conhecidas como ondas Alfvén. Face ao seu papel no processo de transporte de energia e momento, elas são de grande importância na Astrofísica, como será visto adiante. A Fig. 3 ilustra a propagação de uma onda Alfvén.

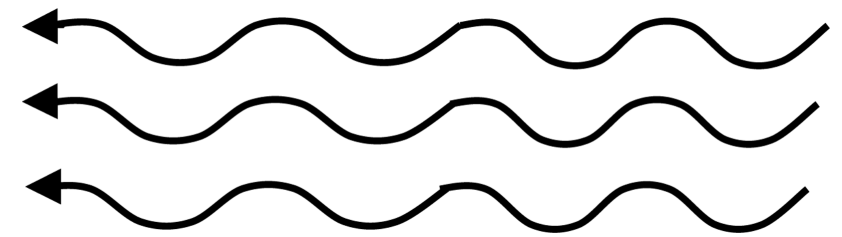

Figura 3 - Propagação de uma onda Alfvén. Representação das linhas de campo magnético congeladas a um plasma com movimento oscilatório.

\subsection{Ondas magnetosônicas}

Onda magnetosônica ou onda magnetoacústica é outro tipo de onda, uma oscilação longitudinal que se observa em plasmas magnetizados. Essas ondas, como o próprio nome deixa transparecer, estão intimamente relacionadas a uma estrutura que se assemelha a uma onda sonora, mas a presença do campo magnético lhe confere algo de especial. De fato, conforme elas se propagam, vão criando regiões de compressão e rarefações não só de densidade do fluido, mas também de intensidade do campo magnético.

Uma maneira sucinta de compreender esse tipo de oscilação pode ser feita retomando a expressão da velocidade de uma onda sonora, Eq. (एб) e, nessa equação, corrigir o valor da pressão, adicionando o termo referente à pressão magnética [73], $P_{M}=B_{0}^{2} / 8 \pi$. Portanto, a pressão, agora corrigida, será uma pressão total, $p_{T}=p+P_{M}$. Designaremos a nova velocidade, velocidade da onda magnetosônica, por $V_{M}$. Então, podemos escrever

$$
V_{M}^{2}=\frac{d}{d \rho}\left(p+\frac{B^{2}}{8 \pi}\right)_{\rho=\rho_{0}},
$$

ou, ainda,

$$
V_{M}^{2}=V_{S}^{2}+\frac{d}{d \rho}\left(\frac{B^{2}}{8 \pi}\right)_{\rho=\rho_{0}},
$$

onde o índice zero refere-se ao estado não perturbado e $V_{S}$ é a velocidade adiabática do som, discutida anteriormente Eqs. (미) e (四).

Nesse ponto, vale salientar qual deve ser a relação entre os valores perturbados e não perturbados das grandezas densidade do fluido e intensidade do campo magnético. Essa relação pode ser facilmente obtida considerando que, para uma coluna do fluido (com a área da base perpendicular às linhas de campo), durante as oscilações provocadas pela onda, as linhas de campo magnético estejam congeladas ao fluido condutor; e que o fluxo de campo magnético (através da base da referida coluna) e o fluxo de massa por unidade de comprimento da coluna sejam conservados.

Assim, podemos escrever

$$
B / \rho=B_{0} / \rho_{0} .
$$

Usando a Eq. (5.) na Eq. (Bप) e o valor da velocidade Alfvén, dado pela Eq. ([2), tem-se 


$$
V_{M}^{2}=V_{S}^{2}+V_{A}^{2}
$$

Da Eq. (\$6), pode-se verificar que, dependendo da relação entre a pressão magnética e a pressão do fluido, a velocidade $V_{M}$ pode ser aproximadamente a velocidade da onda acústica ou a velocidade de uma onda Alfvén. Em outras palavras, o jogo entre pressão magnética e pressão do fluido determina se a velocidade da onda magnetosônica se aproximará da velocidade da onda acústica (no caso do efeito magnético ser desprezível, portanto, pequena pressão magnética) ou se, essencialmente, a velocidade será igual a de uma onda Alfvén (quando o termo de pressão magnética domina o termo de pressão do fluido).

A Fig. 4 ilustra uma onda magnetosônica.

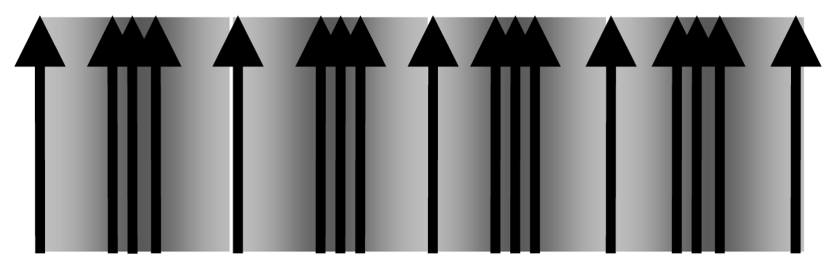

Figura 4 - Onda magnetosônica propagando-se paralelamente ao campo magnético. Observemos as compressões e rarefações de linhas de força e do fluido condutor. Fluido e linhas de força movem-se juntos (situação de congelamento do plasma).

Nossa abordagem explicitou os processos ondulatórios, num plasma magnetizado, utilizando o poder da analogia como instrumento didático. Muito embora tal estratégia seja conveniente para uma primeira apresentação desse conteúdo, enriqueceremos nossa discussão dando, agora, o tratamento mais geral oriundo da magnetohidrodinâmica. Nesse tratamento, os processos de amortecimento das ondas, pelo meio magnetizado, ficarão bem explicitados e, portanto, teremos uma compreensão de como se dá a transferência de energias da onda para o ambiente em que elas se propagam.

\section{Ondas magnetohidrodinâmicas amortecimentos}

\subsection{Introdução}

A abordagem matemática oriunda da teoria MHD permitirá o reconhecimento dos diversos tipos de processos oscilatórios anteriormente tratados numa linguagem menos formal. Na perspectiva de oferecer uma apresentação mais didática, primeiramente as equações apresentadas serão aquelas compatíveis com um fluido condutor perfeito, compressível, não viscoso, que se encontre imerso num campo magnético. Esse nível de simplificação facilitará o reconhecimento das características das ondas geradas no plasma magnetizado, já descritas. A Eq. (5. (5) centralizará toda a discussão, permitindo ainda, com algumas modificações, ampliar o tratamento para um fluido condutor mais realista, onde processos envolvendo viscosidades encontram-se presentes e, portanto, atenuações das ondas poderão ser apreciadas. À medida que parâmetros decisivos para o processo de amortecimento das ondas sejam explicitados, um ganho adicional na compreensão desses processos oscilatórios advirá.

\subsection{Tratamento MHD}

As equações abaixo são pertinentes para a descrição de um fluido condutor perfeito, compressível, não viscoso, imerso num campo magnético. A primeira, a equação da continuidade, é uma expressão da lei da conservação da massa; a segunda, equação de Euler, é a expressão da lei do movimento; e a terceira, de importância central na MHD, é a equação de indução sem o termo difusivo

$$
\begin{gathered}
\frac{\partial \rho}{\partial t}+\nabla \cdot(\rho \mathbf{v})=0 \\
\rho \frac{\partial \mathbf{v}}{\partial t}+\rho(\mathbf{v} \cdot \nabla) \mathbf{v}=-\nabla p-\frac{1}{4 \pi} \mathbf{B} \times(\nabla \times \mathbf{B}), \\
\frac{\partial \mathbf{B}}{\partial t}=\nabla \times(\mathbf{v} \times \mathbf{B}) .
\end{gathered}
$$

Nas condições de equilíbrio, o fluido será tratado como um meio uniforme, em repouso, com densidade uniforme, $\rho_{0}$, imerso num campo magnético uniforme e constante, $\mathbf{B}_{0}$. Uma pequena perturbação nesse meio pode ser imaginada com afastamentos de pequena amplitude dos valores de equilíbrio, a saber

$$
\begin{gathered}
\mathbf{B}=\mathbf{B}_{0}+\mathbf{B}_{1}(\mathbf{x}, t), \\
\rho=\rho_{0}+\rho_{1}(\mathbf{x}, t), \\
\mathbf{v}=\mathbf{v}_{1}(\mathbf{x}, t) .
\end{gathered}
$$

Substituindo-se as Eqs. (四), (四), (स2) nas Eqs. básicas (B7), (Bष), (Bप]) que descrevem o fluido, obtémse, após linearização nos pequenos desvios do equilíbrio, as seguintes equações

$$
\begin{gathered}
\frac{\partial \rho_{1}}{\partial t}+\rho_{0} \boldsymbol{\nabla} \cdot\left(\mathbf{v}_{1}\right)=0 \\
\rho_{0} \frac{\partial \mathbf{v}_{1}}{\partial t}+V_{S}^{2} \boldsymbol{\nabla} \rho_{1}+\frac{\mathbf{B}_{0}}{4 \pi} \times\left(\boldsymbol{\nabla} \times \mathbf{B}_{1}\right)=\mathbf{0} \\
\frac{\partial \mathbf{B}_{1}}{\partial t}-\nabla \times\left(\mathbf{v}_{1} \times \mathbf{B}_{0}\right)=\mathbf{0}
\end{gathered}
$$

onde foi utilizado que

$$
\nabla p=V_{S}^{2} \nabla \rho_{1}
$$

Combinando-se as Eqs. (4.3), (4⿴囗十) e (4.5) podemos obter uma equação para $\mathbf{v}_{1}$ derivando-se em relação ao tempo a Eq. (14), cujo resultado contém derivadas temporais das grandezas $\rho_{1}$ e $\mathbf{B}_{1}$. Uma simplificação 
pode ser feita com o auxílio das Eqs. (4:3) e (45). Desse procedimento, obtém-se

$$
\begin{array}{r}
\frac{\partial^{2} \mathbf{v}_{1}}{\partial t^{2}}-V_{S}^{2} \boldsymbol{\nabla}\left(\boldsymbol{\nabla} \cdot \mathbf{v}_{1}\right)+ \\
\left(\frac{\mathbf{B}_{0}}{4 \pi \rho_{0}}\right) \times \boldsymbol{\nabla} \times\left[\boldsymbol{\nabla} \times\left(\mathbf{v}_{1} \times \mathbf{B}_{0}\right)\right]=\mathbf{0}
\end{array}
$$

Com o auxílio da Eq. (उי), o vetor velocidade Alfvén pode ser definido como sendo

$$
\mathbf{V}_{A}=\mathbf{B}_{0} / \sqrt{4 \pi \rho_{0}} .
$$

Usando a Eq. (48) na Eq. (47), teremos

$$
\begin{array}{r}
\frac{\partial^{2} \mathbf{v}_{1}}{\partial t^{2}}-V_{S}^{2} \boldsymbol{\nabla}\left(\boldsymbol{\nabla} \cdot \mathbf{v}_{1}\right)+ \\
\mathbf{V}_{A} \times \boldsymbol{\nabla} \times\left[\boldsymbol{\nabla} \times\left(\mathbf{v}_{1} \times \mathbf{V}_{A}\right)\right]=\mathbf{0}
\end{array}
$$

A análise das perturbações aqui retratadas é linear. Face à validade do princípio da superposição, neste contexto, qualquer perturbação arbitrária pode ser decomposta em componentes de Fourier. Para uma componente de Fourier típica,

$$
\mathbf{v}_{1}(\mathbf{x}, t)=\mathbf{v}_{1} \exp (i \mathbf{k} \cdot \mathbf{x}-i \omega t),
$$

em que todas as quantidades perturbadas variam com $\exp (i \mathbf{k} \cdot \mathbf{x}-i \omega t)$, solução tipo onda plana permite trocar o operador derivada parcial temporal e o operador nabla com o procedimento

$$
\begin{gathered}
\partial / \partial t \rightarrow(-i \omega), \\
\nabla \rightarrow(i \mathbf{k}) .
\end{gathered}
$$

Assim, a Eq. (4⿴囗), em que foi efetuada a troca dos operadores derivada temporal e nabla, como estabelecido nas Eqs. (5]) e (52), fica

$$
\begin{gathered}
-\omega^{2} \mathbf{v}_{1}+V_{S}^{2}\left(\mathbf{k} \cdot \mathbf{v}_{1}\right) \mathbf{k}- \\
\mathbf{V}_{A} \times\left\{\mathbf{k} \times\left[\mathbf{k} \times\left(\mathbf{v}_{1} \times \mathbf{V}_{A}\right)\right]\right\}=\mathbf{0} .
\end{gathered}
$$

Usando a identidade vetorial abaixo,

$$
\left[\mathbf{k} \times\left(\mathbf{v}_{1} \times \mathbf{V}_{A}\right)\right]=\left(\mathbf{k} \cdot \mathbf{V}_{A}\right) \mathbf{v}_{1}-\left(\mathbf{k} \cdot \mathbf{v}_{1}\right) \mathbf{V}_{A},
$$

na Eq. (5.3), obtém-se

$$
\begin{gathered}
-\omega^{2} \mathbf{v}_{1}+\left(V_{S}^{2}+V_{A}^{2}\right)\left(\mathbf{k} \cdot \mathbf{v}_{1}\right) \mathbf{k}+ \\
\mathbf{V}_{A} \cdot \mathbf{k}\left[\left(\mathbf{V}_{A} \cdot \mathbf{k}\right) \mathbf{v}_{1}-\left(\mathbf{V}_{A} \cdot \mathbf{v}_{1}\right) \mathbf{k}-\left(\mathbf{k} \cdot \mathbf{v}_{1}\right) \mathbf{V}_{A}\right]=\mathbf{0}(55
\end{gathered}
$$

Essa última equação, equação de dispersão para ondas hidromagnéticas, norteará toda a discussão subsequente.
Particularmente, a análise das situações que seguem enriquecerá nossa compreensão dos processos oscilatórios capazes de serem mantidos por um fluido condutor magnetizado.

Assim, convém analisar as situações:

- Para $\mathbf{k} \perp \mathbf{B}_{0}\left(\mathbf{k} \cdot \mathbf{V}_{A}=0\right)$, obtêm-se ondas magnetosônicas.

- Para $\mathbf{k} / / \mathbf{B}_{0}\left(\mathbf{k} \cdot \mathbf{V}_{A}=k V_{A}\right)$, obtêm-se:

- ondas sonoras se $\mathbf{v}_{1}$ for paralelo a $\mathbf{B}_{0}$ e a $\mathbf{k}$.

- ondas Alfvén se $\mathbf{v}_{1}$ for perpendicular a $\mathbf{B}_{0} \mathrm{e}$ a $\mathbf{k}$.

- Para $\mathbf{k}$ formando um ângulo arbitrário com $\mathbf{B}_{0}$, obtêm-se:

- ondas Alfvén puras.

- ondas MHD: rápidas e lentas.

4.2.1. Análise das situações anteriormente mencionadas para obtenção dos diversos tipos de ondas

- Para $\mathbf{k} \perp \mathbf{B}_{0}\left(\mathbf{k} \cdot \mathbf{V}_{A}=0\right)$

A Eq. (5.) fica reduzida

$$
-\omega^{2} \mathbf{v}_{1}+\left(V_{S}^{2}+V_{A}^{2}\right)\left(\mathbf{k} \cdot \mathbf{v}_{1}\right) \mathbf{k}=\mathbf{0}
$$

Portanto, teremos

$$
\mathbf{v}_{1}=\left[\frac{\left(V_{S}^{2}+V_{A}^{2}\right)\left(\mathbf{k} \cdot \mathbf{v}_{1}\right)}{\omega^{2}}\right] \mathbf{k} .
$$

Assim, $\mathbf{v}_{1} / / \mathbf{k}$, ou seja,

$$
\frac{\mathbf{v}_{1}}{v_{1}}=\frac{\mathbf{k}}{k} \text {. }
$$

Substituindo a Eq. (58) na Eq. (57), decorre

$$
\frac{\omega}{k}=\left(V_{S}^{2}+V_{A}^{2}\right)^{\frac{1}{2}} \text {. }
$$

Como visto, temos uma onda com velocidade de fase independente da frequência, onda não dispersiva, e reconhecemos tratar-se de uma onda magnetosônica.

- $\mathbf{k} / / \mathbf{B}_{0} \Rightarrow \mathbf{k} / / \mathbf{V}_{A}$

Dessa condição, decorre que

$$
\frac{\mathbf{V}_{A}}{V_{A}}=\frac{\mathbf{k}}{k} \text {. }
$$

Usando a Eq. (6ل1) na Eq. (5.5), obtém-se

$$
\begin{array}{r}
\left(k^{2} V_{A}^{2}-\omega^{2}\right) \mathbf{v}_{1}+ \\
{\left[\left(V_{S}^{2}+V_{A}^{2}\right)\left(\mathbf{k} \cdot \mathbf{v}_{1}\right)-k V_{A}\left(\mathbf{V}_{A} \cdot \mathbf{v}_{1}\right)\right] \frac{k}{V_{A}} \mathbf{V}_{A}-} \\
k V_{A}\left(\mathbf{k} \cdot \mathbf{v}_{1}\right) \mathbf{V}_{A}=\mathbf{0} .
\end{array}
$$

A manipulação dessa equação conduz a 


$$
\begin{array}{r}
\left(k^{2} V_{A}^{2}-\omega^{2}\right) \mathbf{v}_{1}+ \\
k^{2}\left[\left(\frac{V_{S}^{2}}{V_{A}^{2}}-1\right)\left(\mathbf{V}_{A} \cdot \mathbf{v}_{1}\right)\right] \mathbf{V}_{A}=\mathbf{0} .
\end{array}
$$

Nesse contexto, $\mathbf{k} / / \mathbf{B}_{0}$, verificam-se na Eq. (6²) duas situações de particular interesse, a saber

- $\mathbf{v}_{1} / / \mathbf{V}_{A}$,ou seja, $\mathbf{v}_{1} / / \mathbf{B}_{0}$.

e

- $\mathbf{v}_{1} \perp \mathbf{V}_{A}$, ou seja, $\mathbf{v}_{1} \perp \mathbf{B}_{0}$.

$\mathrm{Na}$ primeira situação, devido aos vetores $\mathbf{v}_{1}$ e $\mathbf{V}_{A}$ serem paralelos, vale a relação

$$
\frac{\mathbf{V}_{A}}{V_{A}}=\frac{\mathbf{v}_{1}}{v_{1}} .
$$

Usando a Eq. ([6.3) na Eq. ([2]), obtém-se

$$
\omega^{2}=k^{2} V_{S}^{2} .
$$

Portanto, resultou em uma onda sonora

$$
\frac{\omega}{k}=V_{S}
$$

A segunda situação, devido aos vetores $\mathbf{v}_{1}$ e $\mathbf{V}_{A}$ serem perpendiculares, é correta a afirmação $\mathbf{v}_{1}$ perpendicular a $\mathbf{B}_{0}$.

Logo,

$$
\mathbf{v}_{1} \cdot \mathbf{V}_{A}=0 .
$$

Assim, a Eq. (52) fica reduzida a

$$
\left(k^{2} V_{A}^{2}-\omega^{2}\right) \mathbf{v}_{1}=\mathbf{0} .
$$

Obtém-se, portanto, uma onda Alfvén

$$
\frac{\omega}{k}=V_{A} .
$$

Uma vez que a velocidade independe da frequência, não há dispersão. Um passo adicional a ser dado é verificar que o campo magnético, associado com a onda Alfvén, está na mesma direção, mas com sentido contrário ao da velocidade $\mathbf{v}_{1}$. Com auxílio da Eq. (5.5) e, considerando que

$$
\mathbf{B}_{1}(\mathbf{x}, t)=\mathbf{B}_{1} \exp (i \mathbf{k} \cdot \mathbf{x}-i \omega t),
$$

obtemos, após a utilização das Eq. (5]) e (52) na (4⿴囗十), a relação

$$
\omega \mathbf{B}_{1}+\mathbf{k} \times\left(\mathbf{v}_{1} \times \mathbf{B}_{0}\right)=\mathbf{0} .
$$

Usando a identidade vetorial abaixo na Eq. (미) e, considerando-se $\mathbf{k} / / \mathbf{B}_{0}$ e $\mathbf{k} \perp \mathbf{v}_{1}$, encontramos a Eq. ([יव)

$$
\begin{gathered}
\mathbf{k} \times\left(\mathbf{v}_{1} \times \mathbf{B}_{0}\right)=\left(\mathbf{k} \cdot \mathbf{B}_{0}\right) \mathbf{v}_{1}-\left(\mathbf{k} \cdot \mathbf{v}_{1}\right) \mathbf{B}_{0}, \\
\mathbf{B}_{1}=-\frac{B_{0}}{\omega / k} \mathbf{v}_{1} .
\end{gathered}
$$

Portanto, $\mathbf{B}_{1}$ é perpendicular ao campo original $\mathbf{B}_{0}$.

Essa pequena componente $\mathbf{B}_{1}$, ilustrada na Fig. 5, quando adicionada ao campo original $\mathbf{B}_{0}$, dá origem às ondulações senoidais nas linhas de campo magnético.

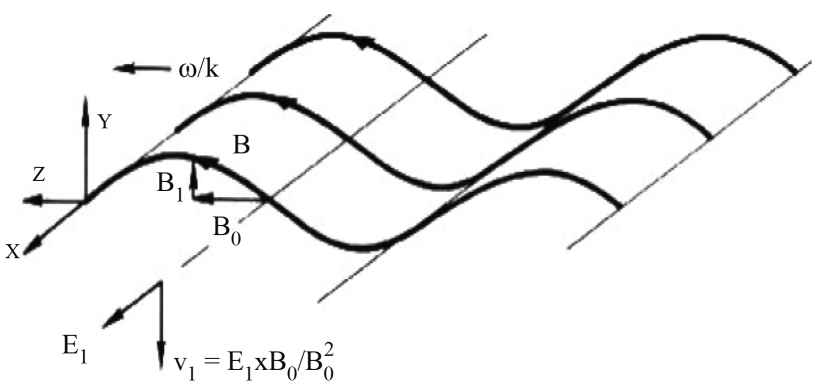

Figura 5 - Onda Alfvén. Fonte: Www.fis.Unb.br/plasma/ Fenomenos $\mathrm{htm}$.

Nesse ponto, podemos calcular densidade de energia magnética $u_{\text {mag. }}$ da onda com auxílio da Eq. ([י2)

$$
u_{\text {mag. }}=\frac{\mathbf{B}_{1} \cdot \mathbf{B}_{1}}{8 \pi}=\frac{B_{0}^{2} v_{1}^{2}}{8 \pi V_{A}^{2}} .
$$

Usando a Eq. ([2) na Eq. ([י]), tem-se

$$
u_{\text {mag. }}=\frac{1}{2} \rho_{0} v_{1}^{2}
$$

ou seja, as densidades de energias magnética e cinética são iguais.

- $\mathbf{k}$ formando um ângulo arbitrário com $\mathbf{B}_{0}$.

A análise dessa situação torna-se matematicamente mais fácil, considerando-se a direção do campo magnético $\mathbf{B}_{0}$ como sendo a direção do eixo cartesiano z e escolhendo o plano xz como sendo aquele que contém o vetor $\mathbf{k}$. Seja $\theta$ o ângulo arbitrário entre o vetor $\mathbf{k}$ e o vetor campo magnético $\mathbf{B}_{0}$.

Embora essas considerações facilitem o tratamento matemático, elas não restringem a generalidade dos resultados; sendo assim, decorre que

$$
\begin{aligned}
\mathbf{B}_{0} & =B_{0} \hat{z}, \\
\mathbf{V}_{A} & =V_{A} \hat{z},
\end{aligned}
$$

$$
\begin{gathered}
\mathbf{k}=k(\hat{x} \sin \theta+\hat{z} \cos \theta) \\
\mathbf{v}_{1}=v_{1 x} \hat{x}+v_{1 y} \hat{y}+v_{1 z} \hat{z} \\
\mathbf{k} \cdot \mathbf{V}_{A}=k V_{A} \cos \theta
\end{gathered}
$$

$$
\mathbf{k} \cdot \mathbf{v}_{1}=k\left(v_{1 x} \sin \theta+v_{1 z} \cos \theta\right)
$$

$$
\mathbf{V}_{A} \cdot \mathbf{v}_{1}=V_{A} v_{1 z},
$$


$\hat{x}, \hat{y}$ e $\hat{z}$ são os vetores unitários nas direções $x, y$ e $z$ respectivamente.

Substituindo-se as Eqs. (76-81) na Eq. (5.9), obtémse

$$
\begin{array}{r}
\mathbf{0}=-\omega^{2}\left(v_{1 x} \hat{x}+v_{1 y} \hat{y}+v_{1 z} \hat{z}\right)+ \\
\left(V_{S}^{2}+V_{A}^{2}\right) k^{2}\left(v_{1 x} \sin \theta+v_{1 z} \cos \theta\right) . \\
(\hat{x} \sin \theta+\hat{z} \cos \theta)+ \\
k V_{A} \cos \theta\left[k V_{A} \cos \theta\left(v_{1 x} \hat{x}+v_{1 y} \hat{y}+v_{1 z} \hat{z}\right)-\right. \\
V_{A} v_{1 z} k(\hat{x} \sin \theta+\hat{z} \cos \theta)- \\
\left.k\left(v_{1 x} \sin \theta+v_{1 z} \cos \theta\right) V_{A} \hat{z}\right] .
\end{array}
$$

- Para a componente $x$ da Eq. (区2), obtém-se

$$
\begin{array}{r}
v_{1 x}\left(-\omega^{2}+k^{2} V_{A}^{2}+k^{2} V_{S}^{2} \sin ^{2} \theta\right)+ \\
v_{1 z}\left(k^{2} V_{S}^{2} \sin \theta \cos \theta\right)=0 .
\end{array}
$$

- Para a componente y da Eq. (区2), obtém-se

$$
v_{1 y}\left(-\omega^{2}+k^{2} V_{A}^{2} \cos ^{2} \theta\right)=0 .
$$

- Para a componente $z$ da Eq. (অ), obtém-se

$$
v_{1 x}\left(V_{S}^{2} k^{2} \sin \theta \cos \theta\right)+v_{1 z}\left(-\omega^{2}+V_{S}^{2} k^{2} \cos ^{2} \theta\right)=0
$$

A análise da componente y, Eq. (B4), revela a existência de uma onda linearmente polarizada, envolvendo oscilações perpendiculares a ke a $\mathbf{B}_{0}$, ou seja, $\left(v_{1 y} \neq 0\right)$. Conclui-se ainda que

$$
-\omega^{2}+k^{2} V_{A}^{2} \cos ^{2} \theta=0 .
$$

Portanto, a velocidade de fase será dada por

$$
\frac{\omega}{k}=V_{A} \cos \theta
$$

logo, trata-se de uma onda Alfvén pura. Observe que a propagação ao longo do campo magnético $(\theta=0)$ dá $\omega / k=V_{A}$, enquanto na propagação perpendicular ao campo magnetostático $\left(\theta=90^{\circ}\right)$, a onda desaparece.

As componentes $x$ e $z$ dão origem a um sistema de duas equações para as amplitudes $v_{1 x}$ e $v_{1 z}$. Para ter solução diferente da trivial $\left(v_{1 x} \neq 0\right.$ e $\left.v_{1 z} \neq 0\right)$, o determinante dos coeficientes desse sistema deve ser nulo.

O cálculo desse determinante fornece

$$
\frac{\omega^{4}}{k^{4}}-\frac{\omega^{2}}{k^{2}}\left(V_{S}^{2}+V_{A}^{2}\right)+V_{A}^{2} V_{S}^{2} \cos ^{2} \theta=0 .
$$

Resolvendo essa equação para $\omega^{2} / k^{2}$, surgem duas soluções reais, a saber

$$
\begin{array}{r}
\frac{\omega^{2}}{k^{2}}=\frac{1}{2}\left(V_{S}^{2}+V_{A}^{2}\right) \pm \\
\frac{1}{2}\left[\left(V_{S}^{2}+V_{A}^{2}\right)^{2}-4 V_{S}^{2} V_{A}^{2} \cos ^{2} \theta\right]^{\frac{1}{2}} .
\end{array}
$$

- Na Eq. (\$प) pode-se verificar que uma das soluções, a com sinal $(+)$, dá um valor para a velocidade de fase maior do que a outra, aquela com o sinal (-). A solução para a equação com o sinal positivo é conhecida como onda $M H D$ rápida. A outra solução com o sinal (-) é chamada de

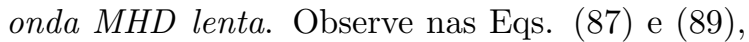
referentes às ondas Alfvén puras, MHD rápida e MHD lenta, que estas ondas não possuem dependência com a frequência, portanto, não apresentam dispersão. A partir da análise da Eq. (区प), podemos evidenciar que a dependência com o valor de $\theta$ pode, por exemplo, conduzir ao desaparecimento de ondas Alfvén puras e de ondas MHD (como no caso de $\theta=90^{\circ}$ ), ou ainda, por exemplo, a ondas MHD rápidas se transformando em magnetosônicas, etc.

\section{Amortecimento das ondas MHD}

Quando o fluido magnetizado não é perfeitamente condutor, ou se efeitos viscosos estão presentes, as ondas que nele se propagam serão amortecidas e suas energias transmitidas para o meio em que estão se propagando. As Eq. (4.3), (44) e (4.5), anteriormente analisadas, precisam ser modificadas para incluir termos que contemplem a natureza viscosa do meio, ou seja,

$$
\frac{\partial \rho_{1}}{\partial t}+\rho_{0} \boldsymbol{\nabla} \cdot\left(\mathbf{v}_{1}\right)=0
$$

$$
\begin{array}{r}
\rho_{0} \frac{\partial \mathbf{v}_{1}}{\partial t}+V_{S}^{2} \nabla \rho_{1}+ \\
\frac{\mathbf{B}_{0}}{4 \pi} \times\left(\nabla \times \mathbf{B}_{1}\right)-\eta_{k} \nabla^{2} \mathbf{v}_{1}=\mathbf{0}
\end{array}
$$

$$
\frac{\partial \mathbf{B}_{1}}{\partial t}-\nabla \times\left(\mathbf{v}_{1} \times \mathbf{B}_{0}\right)-\eta_{m} \nabla^{2} \mathbf{B}_{1}=\mathbf{0},
$$

onde $\eta_{k}$ e $\eta_{m}$ são, respectivamente, a viscosidade e a viscosidade magnética. Para solução tipo ondas planas, pode-se usar as equivalências dos operadores derivada

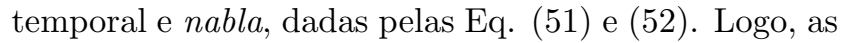
equações linearizadas assumirão as formas

$$
\rho_{1}=\frac{\rho_{0}}{\omega} \mathbf{k} \cdot \mathbf{v}_{1}
$$

$$
\begin{gathered}
\omega \mathbf{v}_{1}=V_{S}^{2} \frac{\rho_{1}}{\rho_{0}} \mathbf{k}+\frac{\mathbf{B}_{0} \times\left(\mathbf{k} \times \mathbf{B}_{1}\right)}{4 \pi \rho_{0}}-\frac{i k^{2} \eta_{k}}{\rho_{0}} \mathbf{v}_{1}, \\
\mathbf{B}_{1}=-\frac{\mathbf{k} \times\left(\mathbf{v}_{1} \times \mathbf{B}_{0}\right)}{\omega+i k^{2} \eta_{m}} .
\end{gathered}
$$

Substituindo-se a Eq. (4.3) na Eq. (प4), encontra-se 


$$
\omega \mathbf{v}_{1}=V_{S}^{2} \frac{\mathbf{k} \cdot \mathbf{v}_{1}}{\omega} \mathbf{k}+\frac{\mathbf{B}_{0} \times\left(\mathbf{k} \times \mathbf{B}_{1}\right)}{4 \pi \rho_{0}}-\frac{i k^{2} \eta_{k}}{\rho_{0}} \mathbf{v}_{1} .
$$

Usando a Eq. (4.5) na Eq. (四), tem-se

$$
\begin{array}{r}
\omega \mathbf{v}_{1}=\frac{V_{S}^{2}}{\omega}\left(\mathbf{k} \cdot \mathbf{v}_{1}\right) \mathbf{k}+ \\
-\frac{\mathbf{B}_{0} \times\left\{\mathbf{k} \times\left[\mathbf{k} \times\left(\mathbf{v}_{1} \times \mathbf{B}_{0}\right)\right]\right\}}{4 \pi \rho_{0}\left(\omega+i k^{2} \eta_{m}\right)}-\frac{i k^{2} \eta_{m}}{\rho_{0}} \mathbf{v}_{1} .
\end{array}
$$

Da Eq. (48), pode-se expressar o vetor campo magnético em função do velocidade Alfvén, ou seja,

$$
\mathbf{B}_{0}=\sqrt{4 \pi \rho_{0}} \mathbf{V}_{A}
$$

Usando-se a Eq. (48) na Eq. (प]), obtém-se

$$
\begin{array}{r}
-\omega^{2}\left(1+\frac{i k^{2} \eta_{m}}{\omega}\right)\left(1+\frac{i k^{2} \eta_{k}}{\omega \rho_{0}}\right) \mathbf{v}_{1}+ \\
V_{S}^{2}\left(\mathbf{k} \cdot \mathbf{v}_{1}\right)\left(1+\frac{i k^{2} \eta_{m}}{\omega}\right) \mathbf{k}- \\
\mathbf{V}_{A} \times\left\{\mathbf{k} \times\left[\mathbf{k} \times\left(\mathbf{v}_{1} \times \mathbf{V}_{A}\right)\right]\right\}=\mathbf{0} .
\end{array}
$$

A comparação entre as Eqs. (4⿴囗十) e (5.3) é inevitável.

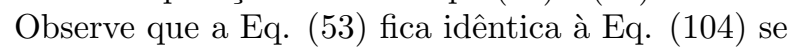

- $\omega^{2}$ for multiplicado pelo fator $\left(1+\frac{i k^{2} \eta_{m}}{\omega}\right) \times$ $\left(1+\frac{i k^{2} \eta_{k}}{\omega \rho_{0}}\right)$

- $V_{S}^{2}$ for multiplicado por $\left(1+\frac{i k^{2} \eta_{m}}{\omega}\right)$.

A partir dessas considerações, podemos inferir as mudanças que estarão sujeitas às velocidades das ondas Alfvén, magnetosônica e sonora. Basta, portanto, quando se fizer necessário, fazer as correções sugeridas acima para o $\omega^{2}$ e para $V_{S}^{2}$. Assim sendo, temos:

- no caso das ondas Alfvén:

para ondas Alfvén transversas (propagando ao longo de $\mathbf{B}_{0}$, a velocidade de fase dada pela Eq. ( $\left.\mathbf{6 8}\right)$ sofre correção inerente ao termo $\omega^{2}$, mencionada anteriormente.

Assim,

$$
\frac{\omega^{2}}{k^{2}}=V_{A}^{2} \Rightarrow \frac{\omega^{2}}{k^{2}}\left(1+\frac{i k^{2} \eta_{m}}{\omega}\right)\left(1+\frac{i k^{2} \eta_{k}}{\omega \rho_{0}}\right)=V_{A}^{2} .
$$

Decorre, portanto, que

$$
k^{2} V_{A}^{2}=\omega^{2}\left[1+i \frac{k^{2}}{\omega}\left(\eta_{m}+\frac{\eta_{k}}{\rho_{0}}\right)-\frac{\eta_{m} \eta_{k} k^{4}}{\omega^{2} \rho_{0}}\right] .
$$

Considerando pequenos os termos de correção correspondentes à viscosidade magnética e à viscosidade, pode-se desprezar o último termo da Eq. (미), a saber: $\frac{\eta_{m} \eta_{k} k^{4}}{\omega^{2} \rho_{0}} \approx 0$.

A Eq. (四) fica

$$
k^{2} V_{A}^{2} \approx \omega^{2}\left[1+i \frac{k^{2}}{\omega}\left(\eta_{m}+\frac{\eta_{k}}{\rho_{0}}\right)\right] .
$$

Em primeira ordem, $\omega / k=V_{A}$; portanto, substituindo o valor de $k^{2}$ no lado direito da Eq. (एण2), resulta

$$
k^{2} V_{A}^{2} \approx \omega^{2}\left[1+i \frac{\omega}{V_{A}^{2}}\left(\eta_{m}+\frac{\eta_{k}}{\rho_{0}}\right)\right] .
$$

Esta Eq. (ㅁ.3) pode ser melhor apresentada usando uma expansão binomial para $x<<1$, a saber

$$
(1+x)^{\frac{1}{2}} \approx 1+\frac{x}{2}
$$

Nesta aproximação, a Eq. ([0]3) ficará

$$
\begin{aligned}
& k V_{A} \approx \omega {\left[1+i \frac{\omega}{V_{A}^{2}}\left(\eta_{m}+\frac{\eta_{k}}{\rho_{0}}\right)\right]^{\frac{1}{2}} \approx } \\
& \omega\left[1+i \frac{\omega}{2 V_{A}^{2}}\left(\eta_{m}+\frac{\eta_{k}}{\rho_{0}}\right)\right],
\end{aligned}
$$

originando para $k$ a expressão complexa onde

$$
k \approx \frac{\omega}{V_{A}}+i \frac{\omega^{2}}{2 V_{A}^{3}}\left(\eta_{m}+\frac{\eta_{k}}{\rho_{0}}\right)=\left(k_{r}+i k_{i}\right),
$$

onde

$$
k_{r} \equiv \frac{\omega}{V_{A}}, \quad k_{i} \equiv \frac{\omega^{2}}{2 V_{A}^{3}}\left(\eta_{m}+\frac{\eta_{k}}{\rho_{0}}\right) .
$$

Qual o significado da parte imaginária do número de onda $k$ ? A parte imaginária de $k$ é responsável pelo amortecimento da onda. A verificação dessa resposta é simples. Vejamos, por questão didática, como isto ocorre no caso simples de uma onda propagando-se na direção $z$

$$
\exp (i k z)=\exp \left(-i k_{i} z\right) \exp \left(i k_{r} z\right) .
$$

Essa última expressão mostra uma onda de número de onda $k_{r}$ propagando-se na direção z e, uma vez que $k_{i}>0$, a amplitude decresce exponencialmente.

Portanto, aceita a afirmação de que a parte imaginária de $k$ é responsável pelo amortecimento da onda, podemos concluir da Eq. (W.5) para uma onda Alfvén, que a atenuação tem o comportamento descrito a seguir.

Atenuação das ondas Alfvén:

1. cresce rapidamente com a frequência; 
2. decresce rapidamente com a intensidade do campo magnético;

3. cresce com a viscosidade do fluido e a viscosidade magnética (lembrando que a viscosidade magnética aumenta com a diminuição da condutividade $\sigma$ do fluido, a saber: $\left.\eta_{m}=c^{2} / 4 \pi \sigma\right)$.

- no caso das ondas sonoras:

para as ondas sonoras propagando-se ao longo de $\mathbf{B}_{0}$, a expressão $\frac{\omega^{2}}{k^{2}}=V_{S}^{2}$ deve ser adaptada levando-se também em conta que:

- $\omega^{2}$ deve ser multiplicado pelo fator $\left(1+\frac{i k^{2} \eta_{m}}{\omega}\right) \times$ $\left(1+\frac{i k^{2} \eta_{k}}{\omega \rho_{0}}\right)$

- $V_{S}^{2}$ deve ser deve ser multiplicado por $\left(1+\frac{i k^{2} \eta_{m}}{\omega}\right)$.

Portanto,

$$
k=\frac{\omega}{V_{S}}\left(1+\frac{i k^{2} \eta_{k}}{\omega \rho_{0}}\right)^{\frac{1}{2}} .
$$

Analogamente, termos de correção viscosa e resistividade serão considerados pequenos.

Assim,

$$
\left(1+\frac{i k^{2} \eta_{k}}{\omega \rho_{0}}\right)^{\frac{1}{2}} \approx 1+\frac{i k^{2} \eta_{k}}{2 \omega \rho_{0}}
$$

Logo,

$$
k \approx \frac{\omega}{V_{S}}\left(1+\frac{i k^{2} \eta_{k}}{2 \omega \rho_{0}}\right)=\left(\frac{\omega}{V_{S}}\right)+i\left(\frac{k^{2} \eta_{k}}{2 V_{S} \rho_{0}}\right) .
$$

Em primeira ordem, $k^{2}=\omega^{2} / V_{S}^{2}$; então, a Eq. (एण]) fica

$$
k=\left(\frac{\omega}{V_{S}}\right)+i\left(\frac{\omega^{2} \eta_{k}}{2 V_{S}^{3} \rho_{0}}\right) \equiv k_{r}+i k_{i} .
$$

A análise da Eq. (एण8) revela, através da parte complexa de $k$, que a atenuação das ondas sonoras:

1. cresce rapidamente com a frequência;

2. cresce com o aumento da viscosidade do fluido:

3. decresce com o aumento da velocidade do som.

Para finalizar essa análise dos processos de amortecimentos das ondas, vejamos como se comportam as ondas magnetosônicas.

- no caso das ondas magnetosônicas: seguindo o processo usual, para as ondas magnetosônicas longitudinais, propagando-se transversalmente ao campo magnetostático $\mathbf{B}_{0}$, a expressão anteriormente obtida, $\omega^{2} / k^{2}=V_{S}^{2}+V_{A}^{2}$, também precisa ser adaptada. Assim,

- $\omega^{2}$ deve ser multiplicado pelo fator $\left(1+\frac{i k^{2} \eta_{m}}{\omega}\right) \times$ $\left(1+\frac{i k^{2} \eta_{k}}{\omega \rho_{0}}\right)$

- $V_{S}^{2}$ deve ser multiplicado por $\left(1+\frac{i k^{2} \eta_{m}}{\omega}\right)$.

A Eq. (5.]) irá se transformar na equação

$$
\begin{aligned}
& k^{2} V_{S}^{2}\left(1+\frac{i k^{2} \eta_{m}}{\omega}\right)+k^{2} V_{A}^{2}= \\
& \omega^{2}\left(1+\frac{i k^{2} \eta_{m}}{\omega}\right)\left(1+\frac{i k^{2} \eta_{k}}{\omega \rho_{0}}\right)
\end{aligned}
$$

Para simplificar, a viscosidade e a viscosidades magnética serão consideradas pequenas. Disso decorre que podemos desprezar termos envolvendo $\eta_{m} \eta_{k} k^{4} / \omega^{2}$, ou seja,

$k^{2}\left(V_{S}^{2}+V_{A}^{2}\right)=\omega^{2}\left[1+i \frac{k^{2}}{\omega}\left(\frac{\eta_{k}}{\rho_{0}}+\eta_{m}\right)-i \frac{k^{4} \eta_{m} V_{S}^{2}}{\omega^{3}}\right]$,

ou ainda,

$k=\frac{\omega}{\left(V_{S}^{2}+V_{A}^{2}\right)^{\frac{1}{2}}}\left\{1+i \frac{k^{2}}{\omega}\left[\frac{\eta_{k}}{\rho_{0}}+\eta_{m}\left(1-\frac{k^{2} V_{S}^{2}}{\omega^{2}}\right)\right]\right\}^{\frac{1}{2}}$.

Na Eq. (एण. $)$, podemos trocar $\omega^{2} / k^{2}$ pela apro$\operatorname{ximação~}\left(V_{S}^{2}+V_{A}^{2}\right)$.

Assim,

$$
\begin{array}{r}
k=\frac{\omega}{\left(V_{S}^{2}+V_{A}^{2}\right)^{\frac{1}{2}}} \times \\
\left\{1+i \frac{k^{2}}{\omega}\left\{\frac{\eta_{k}}{\rho_{0}}+\eta_{m}\left[1-V_{S}^{2}\left(V_{S}^{2}+V_{A}^{2}\right)^{-1}\right]\right\}\right\}^{\frac{1}{2}} .
\end{array}
$$

Expandindo o termo entre chaves que se encontra elevado ao fator $1 / 2$, tem-se

$$
\begin{gathered}
\left\{1+i \frac{k^{2}}{\omega}\left\{\frac{\eta_{k}}{\rho_{0}}+\eta_{m}\left[1-V_{S}^{2}\left(V_{S}^{2}+V_{A}^{2}\right)^{-1}\right]\right\}\right\}^{\frac{1}{2}} \approx \\
1+i \frac{k^{2}}{2 \omega}\left\{\frac{\eta_{k}}{\rho_{0}}+\eta_{m}\left[1-V_{S}^{2}\left(V_{S}^{2}+V_{A}^{2}\right)^{-1}\right]\right\} .
\end{gathered}
$$

Substituindo-se a Eq. (ए3) na Eq. (एव), decorre

$$
\begin{array}{r}
k=\frac{\omega}{\left(V_{S}^{2}+V_{A}^{2}\right)^{\frac{1}{2}}}+ \\
i \frac{k^{2}}{2\left(V_{S}^{2}+V_{A}^{2}\right)^{\frac{1}{2}}}\left[\frac{\eta_{k}}{\rho_{0}}+\eta_{m}\left(1-\frac{V_{S}^{2}}{V_{S}^{2}+V_{A}^{2}}\right)\right]
\end{array}
$$


ou ainda, usando a aproximação em primeira ordem, $\omega^{2} / k^{2} \approx V_{A}^{2}+V_{S}^{2}$, obtém-se

$$
k=\frac{\omega}{\left(V_{S}^{2}+V_{A}^{2}\right)^{\frac{1}{2}}}+i \frac{\omega^{2}}{2\left(V_{S}^{2}+V_{A}^{2}\right)^{\frac{3}{2}}}\left[\frac{\eta_{k}}{\rho_{0}}+\frac{\eta_{m}}{\left(1+\frac{V_{S}^{2}}{V_{A}^{2}}\right)}\right] .
$$

Concluímos, portanto, que a atenuação de uma onda magnetosônica tem o seguinte comportamento:

1. cresce com a frequência;

2. cresce com a viscosidade magnética e cinemática $\left(\eta_{k} / \rho_{0}\right)$

3. decresce com o aumento da intensidade do campo magnético.

\section{Comentários finais}

Uma ampla reflexão nos permite concluir que, como anteriormente já ressaltado, provavelmente, o aquecimento da corona estelar não pode estar reduzido a um único processo, contemplando, assim, inúmeros mecanismos ao mesmo tempo. Alguns desses mecanismos podem operar globalmente, enquanto outros apenas em situações físicas particulares. A análise teórica aqui apresentada revela que a presença de campos magnéticos fortes na atmosfera estelar reduz a atenuação das ondas Alfvén e magnetosônicas. Assim, nas situações em que o dínamo estelar seja suficientemente forte para intensificar o campo magnético, decorrerá uma redução na atenuação das ondas Alfvén e magnetosônicas. Menor atenuação dessas ondas implica, então, menor depósito de energia na atmosfera estelar, com consequente enfraquecimento em sua contribuição no processo de aquecimento e produção da atividade coronal.

Uma vez que processos de origem magnética não possam ser os principais agentes do aquecimento, parece claro, para a situação acima descrita, que mecanismo de origem não-magnética se faz presente, sendo, portanto, o depósito de energia na coroa estelar realizado mais fortemente via atenuação de ondas mecânicas [1]. Face às simplificações inerentes ao tratamento dado ao plasma estelar, devemos ter em mente que, nosso desenvolvimento teórico constitui, apenas, uma abordagem introdutória desse complexo processo de aquecimento, com detalhes pouco conhecidos até hoje.

\section{Referências}

[1] O. R. Nelson, Sobre a Natureza dos Processos Físicos de Aquecimento da Atmosfera de Estrelas do Tipo Solar. Tese de Doutorado, Universidade Federal do Rio Grande do Norte, 2008.
[2] W.J. Maciel, Ciclo de Seminários de Iniciação Científica, IAG/USP (abril, 2005).

[3] K.S.O. Filho, Saraiva, M.F.O. Saraiva, Astronomia \& Astrofísica (Editora Livraria da Física, São Paulo,2004), $2^{\text {a }}$ ed.

[4] B. Edlén, Ark. Mat. Astr. Och. Fys. 28, B, 1 (1941).

[5] L. Biermann, Naturwiss 33, 118 (1946).

[6] L. Biermann, Zeitschrift fur Astrophysik 25, 161 (1948).

[7] M. Schwarzschild, Ap J. 107, 1 (1948).

[8] E. Schatzman, Ann. Astrophys. 12, 203 (1949).

[9] H. Schirmer, Zeitschrift fur Astrophysik 27, 132 (1950).

[10] C. De Jager, in: Handbuck der Physik 52 (S.F. Flugge Ed., Berlin, 1959), p. 80.

[11] C. De Jager and M. Kuperus, Bull. Astron. Inst. Neth. 16, 71 (1961).

[12] D.E. Osterbrock, Astrophys. J. 134, 347 (1961).

[13] G.A. Bird, Astrophys. J. 139, 675 (1964a).

[14] G.A. Bird, Astrophys. J. 139, 684 (1964b).

[15] G.A. Bird, Astrophys. J. 141, 1455 (1965).

[16] M. Kuperus, The Transfer of Mechanical Energy in the Sun and the Heating of Corona. Ph. D. Thesis Univ. Utrecht, Netherlands 1965.

[17] M. Kuperus, Space Sci. Rev. 9, 713 (1969).

[18] M. Kuperus, Solar Phys. 22, 257 (1972).

[19] R.A. Kopp, Ph.D. The Equilibrium Structure of a Shock-Heated Corona. PhD. Thesis, Air Force Cambridge Res. Lab. Sci. Rept. 4, Bedford, 1968.

[20] R. Stefanik, Hydrodynamic Phenomena in Variable Stars. Ph.D. Thesis, Harvard University, Cambridge, Massachusetts, USA, 1973.

[21] R.F. Stein, in: Cromosphere-Corona Transition Region, edited by R.G. Athay and G. Newkirk, (Hight Altitude Observatory, Boulder, 1969), p. 171,

[22] R.F. Stein and R.A. Schwartz, The Astrophysical Journal 177, 807 (1972).

[23] G. Gonczi, A. Mangeney and P. Souffrin, Astron. Astrophys. 54, 689 (1977).

[24] P. Ulmschneider, Z. Astrophys. 67, 193 (1967).

[25] P. Ulmschneider, Solar Phys. 12, 403 (1970).

[26] P. Ulmschneider, Astron. Astrophys. 12, 297 (1971a).

[27] P. Ulmschneider, Solar Phys. 39, 327 (1974).

[28] P. Ulmschneider, Solar Phys. 49, 249 (1976).

[29] P. Ulmschneider, Astron. Astrophys. 222, 171 (1989).

[30] H. Afvén, Mon. Not. Roy. Astron. Soc. 107, 211 (1947).

[31] H. C. Van de Hulst, In the sun, edited by G.P. Kuiper (Chicago Univ. Press, Chicago, 1953), p.207.

[32] J.H. Piddington, Mon. Not. Roy. Astron. Soc., 116, 314 (1956).

[33] C. De Jager, Nuovo Cimento Suppl. Ser. 10, 291 (1959a).

[34] H.W. Babcock and H.D. Babcock, International Astronomical Union Symposium n. 6: Eletromagnetic Phenomena in Cosmical Physics, edited by B. Lehnert (Cambridge University Press, Cambridge, 1958), p. 239 . 
[35] R. Howard, Ap. J. 130, 193 (1959).

[36] R.B. Leighton, Ap. J. 130, 366 (1959).

[37] W.O. Roberts, Ap. J. 101, 136 (1945).

[38] O. Mohler, Mon. Not. Roy. Astron. Soc. 111, 630 (1951).

[39] R.N. Thomas, Ap. J. 108, 130 (1948).

[40] D.E. Billings, A Guide to the Solar Corona (Academic Press, New York, 1966).

[41] E. Schatzman and P. Souffrin, Ann. Rev. Astron. Astrophys. 5, 67 (1967).

[42] J.H. Piddington, Solar Phys. 33, 363 (1973).

[43] R.J. Bray and R.E. Loughhead, The Solar Chromosphere (Chapman and Hall, London, 1974).

[44] R.F. Stein and J. Leibacher, Ann. Rev. Astron. Astrophys. 12, 407 (1974).

[45] R.F. Stein and J. Leibacher, in: Stellar Turbulence, edited by D.F. Gray and J.L. Linsky (Springer, Berlin, 1980), p. 225.

[46] R.G. Athay, The Solar Chromosphere and Corona: Quiet Sun (Reidel, Dordrecht, 1976).

[47] G.L. Withbroe and R.W. Noyes, Ann. Rev. Astron. Astrphys. 15, 363 (1977).

[48] D.G. Wentzel, Rev. Geophys. Space Phys. 16, 757 (1978).

[49] D.G. Wentzel, in: Coronal Heating in The Sun as a Star, (Monograph Series on Nonthermal Phenomena in Stellar Atmospheres), edited by S. Jordan (NASA, SP-450, Washington, DC, 1981), p. 331.

[50] R. Mewe, Space Sci. Rev. 24, 101 (1979).

[51] P. Ulmschneider, Space Sci. Rev. 24, 71 (1979).

[52] P. Ulmschneider, in: The solar Phenomena in Stars and Stellar Systems, edited by R.M. Bonnet and A.K. Dupree (Reidel, Dordrecht, 1981), p. 239.

[53] J.W. Leibacher and R.F. Stein, in: Highlights of Astronomy 5, edited by P.A. Wayman (Reidel, Dordrecht, 1980), p. 581.

[54] J.W. Leibacher and R.F. Stein, 1981, in: Second Cambridge Workshop on Cool Stars, Stellar Systems and the Sun, Vol.1, edited by M.S. Giampapa and L. Golub, Smithsonian Special Report $\mathrm{N}_{0}$ 392, p. 23.

[55] C. Chiuderi, in: The Solar Phenomena in Stars and Stellar Systems, edited by R.M. Bonnet and A.K. Dupree (Reidel, Dordrecht, 1981), p. 267.
[56] C. Chiuderi, in: Solar and Magnetic Fields: Origins and Coronal Effects, (IAU Symp. 102, J. O. Stenflo Ed.,p. 375, 1983).

[57] J.V. Hollweg, in: The Solar Active Regions edited by F.Q. Orrall (Colorado Ass. Univ. Press, Boulder, 1981a), p.277.

[58] J.V. Hollweg, Coronal Heating by Waves, In JPL Solar Wind Five, edited by M. Neugebauer (NASA, CP-2280, 1983), p. 5 .

[59] J.V. Hollweg, in: Advances in Space Plasma Physics, edited by B. Buti (World Scientific Publ. Co., Singapore, 1985b), p. 77 .

[60] M. Kuperus, J.A. Ionson and D.S. Spicer, Ann. Rev. Astron. Astrophys. 19, 7 (1981).

[61] E.R. Priest, Solar Magnetohydrodynamics (Reidel, Dordrecht, , 1982).

[62] E.R. Priest, Solar Phys. 86, 33 (1983).

[63] L. Golub, in: Proc. IAU Symp. 102, edited by J.O. Stenflo (Reidel, Dordrecht, 1983) p. 345.

[64] M. Kuperus, Space Sci. Rev. 34, 47 (1983).

[65] J. Heyvaerts, in: The Hydromagnetics of the Sun, (Proceedings of the Fourth European Meeting, 1984).

[66] J. Heyvaerts, in: Unstable Current Systems and Plasma Instabilities in Astrophysics, edited by M.R. Kundu and G.D. Holman (IAU Symp. 107, 1985), p. 95.

[67] J.P. Cassinelli and K.B. MacGregor, Physics of the Sun, Vol. III, edited by P.A. Sturrock, T.E. Holzer, D. Mihalas and R. Ulich (Reidel, Dordrecht, 1986), p. 70.

[68] E.N. Parker, Coronal heating by waves, in: JPL Solar Wind Five, edited by M. Neugebauer (NASA CP 2280, 1983c), p. 23.

[69] E.N. Parker, Heating of the stellar corona, edited by A.I. Poland (NASA CP - 2442, 1986), p. 9.

[70] R. Rosner, B.C. Low, T.E. Holzer, in: Physics of The Sun, Vol II, edited bu P.A. Sturrock (Reidel, Dordrecht, 1986), p. 135.

[71] P. Ulmschneider, Adv. Space Res. 6, 39 (1986).

[72] U. Narain and P. Ulmschneider, Space Science Reviews 54, 377 (1990).

[73] J.A. Bittencourt, Fundamental of Plasma Physics (Editora Abaeté, São Paulo, 1995), $2^{\mathrm{a}}$ ed. 